\title{
Small interfering RNA against PTTG: A novel therapy for ovarian cancer
}

\author{
SHAHENDA M. EL-NAGGAR*, MOHAMMED T. MALIK* and SHAM S. KAKAR \\ Departments of Medicine, Biochemistry and Molecular Biology, and the James Graham \\ Brown Cancer Center, University of Louisville, Louisville, KY 40202, USA
}

Received November 24, 2006; Accepted January 15, 2007

\begin{abstract}
Ovarian epithelial cancer is a significant cause of death among women, accounting for $5 \%$ of all female cancer-related fatalities. A lack of reliable detection methods and resistance to chemotherapy agents are considerable obstacles in the treatment of this cancer. Recently, high-level expression of the pituitary tumor transforming gene (PTTG) was found in a wide range of tumors, including ovarian cancers. Elevated PTTG levels were found to induce cellular transformation in vitro and tumor formation in nude mice. Therefore, we hypothesize a correlation exists between the levels of PTTG expression and tumorigenesis, and that downregulation of PTTG levels will result in the suppression of tumor growth. We used small interfering RNA (siRNA) to silence PTTG expression in human A2780 ovarian carcinoma cells and assessed the effect of PTTG silencing in tumor formation in vitro and in vivo. The siRNA directed against PTTG reduced its expression at both the mRNA and protein levels. A fifty percent reduction in cell proliferation was achieved in cells constitutively expressing PTTG siRNA compared to vector or control-siRNA transfected cells. Furthermore, colony formation in soft agar was reduced by $70 \%$ in PTTG siRNA stable cell lines. Using nude mice, we showed that animals injected with A2780 cells constitutively expressing PTTG-siRNA decreased the incidence of tumor development and tumor growth. Taken together, these results strongly suggest that PTTG may serve as an important molecular target for the discovery of new anticancer agents and treatment strategies.
\end{abstract}

\section{Introduction}

Ovarian cancer continues to be a significant cause of death among women in the western world. With an increasing

Correspondence to: Dr Sham S. Kakar, Department of Medicine, 580 South Preston Street, Baxter II, Room 321E, University of Louisville, Louisville, KY 40202, USA

E-mail: sskaka01@louisville.edu

${ }^{*}$ Contributed equally

Key words: securin, PTTG, tumor growth, ovarian tumor, siRNA number of cases not diagnosed due to a lack of appropriate screening methods or detected at late stages, rising incidence effected approximately 20,000 individuals in 2006. The etiology of ovarian cancer is not clearly understood; however, it is believed that multiple genetic lesions are required to initiate ovarian epithelial cancer. Currently, chemotherapy is among the standard management approaches (1), yet resistance to chemotherapeutic agents is a treatment obstacle for many patients (2). Therefore, a novel therapeutic approach is needed.

Initially cloned from the rat pituitary tumor (3), pituitary tumor transforming gene (PTTG), also known as securin, is an oncogene that is highly expressed in many tumors including pituitary $(4,5)$, lung $(6)$, colon, and ovarian tumors (7-10). On the other hand, PTTG expression in normal tissue is very low except for the testis (8). Sharing the same function as the yeast pds1 (11), securin shares no sequence homology to any other protein identified to date. Based on work from our laboratory and others, securin has been suggested to be a multifunctional protein. Some of its functions include inhibition of sister chromatids separation (11), DNA repair (12), transactivation of other genes (13), and secretion and expression of angiogenic and metastatic factors (14-16). Studies with NIH3T3 $(3,8)$ and HEK-293 cells (16) demonstrated the ability of PTTG to promote cellular transformation in vitro and tumor formation in vivo. Furthermore, PTTG overexpression induces aneuploidy (17) genomic instability (18), and invasiveness (19).

By generating PTTG null mice (PTTG -/-), the physiological function of PTTG in cellular transformation was demonstrated. PTTG -/- mice developed splenic and testicular hypoplasia, thrombocytopenia, aberrant cell cycle progression, and premature centromere division, however, these mice were viable and fertile (20). Furthermore, crossing PTTG -/- with $\mathrm{Rb}$ heterozygous mice $(\mathrm{Rb}+/-)$ resulted in a $30 \%$ pituitary tumors compared to $87 \%$ in PTTG +/+/ Rb (+/-) mice (21). Previous studies with antisense oligonucleotides targeted against PTTG in ovarian tumor cell line (SK-OV3) and cervical tumor cell line (HelaS3) $(22,23)$, were effective at reducing cell proliferation. Recent work with siRNA directed against PTTG in hepatoma cells was successful at reducing tumorigenic phenotypes of these cells in p53-dependent manner (24). Collectively, these data suggest an important role of PTTG in tumorigenesis.

In the present study, we reduced PTTG expression levels using PTTG specific small interfering RNA (siRNA) to 
investigate the consequences of overexpression of PTTG for tumor cell proliferation and growth. The use of siRNA has been a powerful tool used by many researchers to decrease expression or silence target genes. Once introduced into the cell, short 19-21 bp siRNA binds to complementary specific mRNA strand. These short, double-stranded RNA mediate the degradation of complementary mRNA through the recruitment of RNA silencing complex (RISC) (25). Studies in plants and $C$. elegans showed that siRNA can affect gene expression by inducing promoter methylation or by inhibiting protein synthesis (25). Our data suggest that reducing PTTG in ovarian cancer cells A2780 leads to the inhibition of tumor cell proliferation and a lower incidence of tumor development in nude mice.

\section{Materials and methods}

Cell lines and cell culture. Human epithelial ovarian carcinoma cell line (A2780) was obtained from Dr Denise Connolly (Fox Chase Cancer Center, Philadelphia, PA). These cells were cultured in RPMI-1640 (Invitrogen, Carlsbad, CA) supplemented with $10 \%$ fetal bovine serum (Hyclone, Atlanta, GA) and maintained in a humidified atmosphere at $37^{\circ} \mathrm{C}$ and $5 \%$ $\mathrm{CO}_{2}$. Colon cancer cell lines HCT116 (wild-type) and PTTG knockout (PTTG -/-) were obtained from Dr Bert Vogelstein (Johns Hopkins University, Baltimore, MD) and grown according to instructions from the supplier. The cells lines were cultured on a routine basis every 3-4 days.

Generation of plasmid constructs. To generate constructs constitutively expressing PTTG-siRNA or control-siRNA, we cloned PTTG-siRNA or control siRNA into pSUPER.retro. puro (OligoEngine, Seattle, WA) expression plasmid vector. Sequences used were for PTTG sense (5'-GAT CCC CTG GGA GAT CTC AAG TTT CAT CAA GAG ATG AAA CTT GAG ATC TCC CAT TTT TA-3') and antisense (5'-AGC TTC CAAA AAT GGG AGA TCT CAA GTT TCA CTC TTG AAT GAA ACT TGA GAT CTC CCA GGG-3') encoding a siRNA against 19 nucleotides sequences (5'-TGG GAG ATC TCA AGT TTC A-3') of human PTTG. For control sequence, we used two complementary oligonucleotides sense (5'-GAT CCC CAT GTA TTG GCC TGT ATT AGT TCA AGA GAC TAA TAC AGG CCA ATA CAT TTT TTA-3') and antisense (5'-AG CTT CCA AAA AAT GTA TTG GCC TGT ATT AGT CTC TTG AAC TAA TAC AGG CCA ATA CAT GGG-3'). The oligonucleotides were obtained from Invitrogen. The sequences were flanked by BgIII and HindIII restriction enzyme sequences for cloning purpose. Equal amounts of sense and antisense primers were mixed and annealed in a $50-\mu 1$ reaction by heating at $90^{\circ} \mathrm{C}$ for $4 \mathrm{~min}$ followed by $70^{\circ} \mathrm{C}$ for $10 \mathrm{~min}, 60^{\circ} \mathrm{C}$ for $30 \mathrm{~min}, 37^{\circ} \mathrm{C}$ for $20 \mathrm{~min}$, and $25^{\circ} \mathrm{C}$ for $10 \mathrm{~min}$. The pSUPER.retro.puro expression vector was digested with $B g l \mathrm{II}$ and HindIII and purified on agarose gel. The annealed oligos were purified on agarose gel and directionally cloned into pSUPER.retro.puro plasmid vector, which directs the synthesis from the H6 promoter. All plasmid constructs were verified by nucleotide sequence analysis.

Generation of cell lines that express siRNA constructs. To generate stable clones constitutively expressing siRNA, A2780 cells were plated in 6-well plates $24 \mathrm{~h}$ prior to transfection. Cells were transfected with vector alone, control-siRNA, or PTTG-siRNA cloned into pSUPER.retro.puro plasmid (see above) using transfectin transfection reagent (Bio-Rad, Hercules, CA) as described previously (8). Forty-eight hours after transfection, cells were trypsinized and plated in a 100-mm dish. Cells were treated with puromycin (SigmaAldrich Co., St. Louis, MO) $8 \mu \mathrm{g} / \mathrm{ml}$ for 2 weeks, cell lines were selected. Expression of each cell line was determined by Western blot analysis.

Western blot analysis. Cells were cultured in complete growth media to $80-90 \%$ confluence. Cells were washed and collected in cold PBS and lysed with lysis buffer (10 mM Tris- $\mathrm{HCl}$, pH 7.5; $150 \mathrm{mM} \mathrm{NaCl} ; 1 \%$ Triton X-100; 1 mM EDTA). Protein concentration for each sample was assayed by Bradford Method (Bio-Rad Laboratories). Forty micrograms of protein from each sample was resolved on $15 \%$ SDS polyacrylamide gel and transferred to nitrocellulose membranes (GE Healthcare Biosciences, Piscataway, NJ). Membranes were blocked overnight with $5 \%$ non-fat milk/Tween $(20 \mathrm{mM}$ Tris $/ \mathrm{HCl}$, $150 \mathrm{mM} \mathrm{NaCl}, 0.1 \%$ Tween-20, pH 7.6) (TBST). Membranes were incubated with PTTG antiserum diluted at $(1: 1,500)$ (9) for $1 \mathrm{~h}$ at room temperature followed by 3 washes with TBST and then incubated with HRP-conjugated goat anti-rabbit secondary antibody diluted at 1:5,000. The immune complexes formed were detected by ECL reagents (GE Healthcare Biosciences). The blots were stripped and reprobed with $B$-actin antibody (Sigma-Aldrich Co.) to examine equal loading.

Cell proliferation assay. To determine the effect of PTTG down-regulation on cell proliferation, A2780 cells transfected with vector, control siRNA, or PTTG siRNA plasmid were seeded $(2,500$ cells/well $)$ in 96-well plate in triplicate in complete culture growth media containing $8 \mu \mathrm{g} / \mathrm{ml}$ puromycin and incubated overnight at $37^{\circ} \mathrm{C}$. The next day, medium was replaced with growth medium containing $5 \%$ FBS. Cell proliferation was performed daily using a non-radioactive assay system from Promega (Madison, WI). Briefly, after every 24 h, $20 \mu \mathrm{l}$ of MTS reagent (CellTiter $96 \mathrm{AQ}_{\text {ueous }}$ ) was added to each well and incubated at $37^{\circ} \mathrm{C}$ for $1 \mathrm{~h}$. Absorbance was recorded at $490 \mathrm{~nm}$ (8). Experiments were repeated 3 times.

Colony formation assay. To test the effect of PTTG silencing on anchorage independent growth of A2780 cells, cells transfected with vector, control siRNA, or PTTG siRNA plasmid in log phase were trypsinized and counted. Ten thousand cells were mixed with $1.0 \mathrm{ml}$ of $0.33 \%$ agar in complete medium/dish and plated in $35-\mathrm{mm}$ tissue culture dishes layered with $2.0 \mathrm{ml}$ of $0.50 \%$ agar. Cells were treated with fresh growth medium every 4-5 days for 2 weeks until the colonies were formed. Colonies were counted and photographed. Experiments were repeated twice.

Tumor development in nude mice. To assess in vivo activity of PTTG silencing, A2780 cells transfected with control plasmid, control siRNA, or PTTG siRNA were trypsinized, washed twice with PBS, and resuspended in RPMI media without serum and antibiotic. Cells $\left(2 \times 10^{6} /\right.$ site $)$ were injected subcutaneously into both flanks of 4 to 6 -week-old nu/nu 


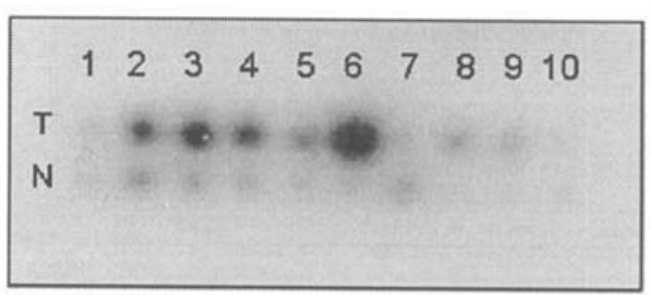

Figure 1. Expression of PTTG mRNA in ovarian tumors. An ovarian cancer profiling array was hybridized with $\left[{ }^{32} \mathrm{P}\right]$-labeled PTTG $\mathrm{cDNA}$. T, tumor and $\mathrm{N}$, normal tissue. T1, papillary serous cystadenoma; T2, leiomyoma; T3, adenocarcinoma; T4, clear cell adenocarcinoma; T5, adenocarcinoma; T6, leiomyoma; T7, mucinous cystadenocarcinoma; T8, adenocarcinoma; T9, serous surface papillary carcinoma; and T10, papillary serous cystadenoma.

mice (NCI). Five mice were used in each group. After 12 days of injection, tumor volumes were recorded every fourth day for 2 weeks (6). For control experiments, mice were injected with colon cancer cell line HCT116 and PTTG knockout HCT116/PTTG (-/-) cell lines. Tumor volumes $\left(\mathrm{mm}^{3}\right)$ were calculated according to the formula: tumor volume $\left(\mathrm{mm}^{3}\right)=($ long dimension $) \mathrm{x}(\text { short dimension })^{2} / 2$. After 4 weeks of injection of cells, animals were sacrificed; tumors were collected, measured, and weighed. One part of each tumor tissue was fixed in buffered formalin and another part was frozen in liquid nitrogen and stored at $-80^{\circ} \mathrm{C}$ for future use. In vivo studies were carried out in accordance with the University of Louisville Institutional Animal Care and Use Committee guidelines.

Statistical analysis. For cell proliferation assay, One-way ANOVA (PRSIM software, version 3.0; Graph pad, San Diego, CA) was performed and the difference between groups was determined by Tukey-Kramer multiple comparison test. For tumor formation in nude mice, the difference between experimental groups was determined by Bonferroni post-test.

\section{Results and Discussion}

Transfection of ovarian tumor A2780 cells with PTTG siRNA down-regulates the expression of PTTG. In our previous studies using Northern blotting, reverse transcriptase/polymerase chain reaction (RT/PCR), and immunohistochemical analysis, we showed high levels of expression of PTTG mRNA and protein in ovarian tumors and tumor derived cell lines (8-10). In the present study, we used an ovarian cancer profiling array (Clontech, Palo Alto, CA) that included normalized cDNA from 10 different ovarian tumors and 10 corresponding normal ovarian tissues, along with negative and positive controls. A high level of PTTG expression was observed in 7 out of 10 tumor tissues compared to normal tissues (Fig. 1). These results confirm our previous results for the high levels of expression of PTTG in most of the ovarian tumors and tumor-derived cell lines. Cloning and sequencing of PTTG from various ovarian tumors showed sequence identity with the human testis cDNA (10), suggesting that the oncogenic function of PTTG is not due to its mutation but rather to its overexpression. Based on these results, we hypothesized that a correlation exists between the level of expression of PTTG and tumorigenesis, and that down-regulation of PTTG
A
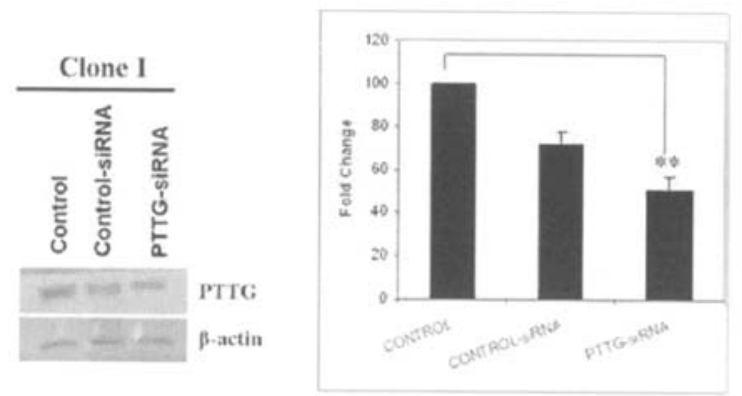

B
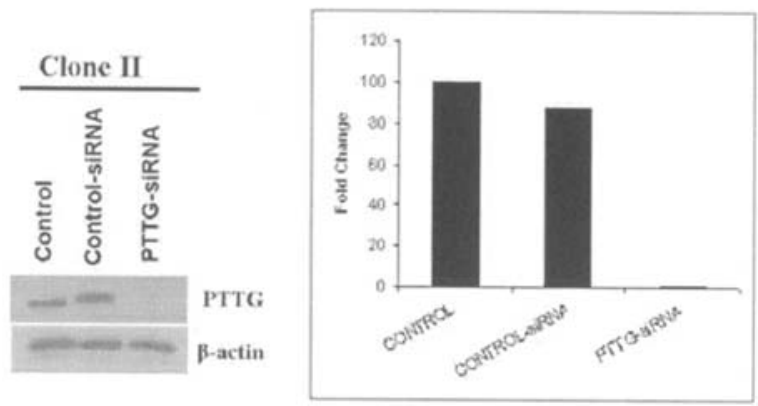

Figure 2. Generation of PTTG siRNA stable cell lines. Ovarian epithelial cells (A2780) were transfected with vector pSUPER.reto.puro (control) control-siRNA.pSUPER.retro.puro (Control-siRNA) or PTTG-siRNA. pSUPER.retro.puro (PTTG-siRNA) plasmid. Forty-eight hours after transfection, cells were diluted and replated and then treated with puromycin $(8 \mu \mathrm{g} / \mathrm{ml})$ for two weeks. Cell lines were selected and expanded. Two cell lines from each were selected. Two cell lines from $P T T G$-siRNA were selected, one that showed partial reduction of PTTG expression (PTTG-siRNA Clone I) and another that showed complete reduction of PTTG expression (PTTG-siRNA Clone II). Western blot analysis was performed using PTTGspecific antibody. $\beta$-actin was used as a loading control. Data shown are representative of three independent experiments.

expression in tumors will lead to suppression of tumor growth and development. In our recent studies, we showed that down-regulation of PTTG in lung tumor cells (H1299) by PTTG siRNA resulted in the inhibition of colony formation in vitro and tumor development and growth in nude mice (6). Consistent with our results, Cho-Rok et al (24), using an adenovirus system expressing PTTG siRNA to downregulate PTTG expression, recently showed a significant reduction in tumor growth in vitro and in vivo for hepatoma cells. Taken together, these results suggest that downregulation of PTTG expression can reduce tumor development and growth. However, use of siRNA is shortlived and the use of adenovirus to deliver siRNA to diminish PTTG expression has limitations. It can infect normal tissues and is accompanied by acute toxicity when used at a high dosage. To overcome this, we generated stable clones constitutively expressing control-siRNA or PTTG-siRNA when transfected with the vector containing PTTG siRNA or control siRNA. We cloned PTTG-siRNA or control-siRNA into pSUPER.retro.puro vector as described in Materials and methods. Positive clones were identified by restriction of the plasmid DNA with EcoRI and HindIII restriction 
A

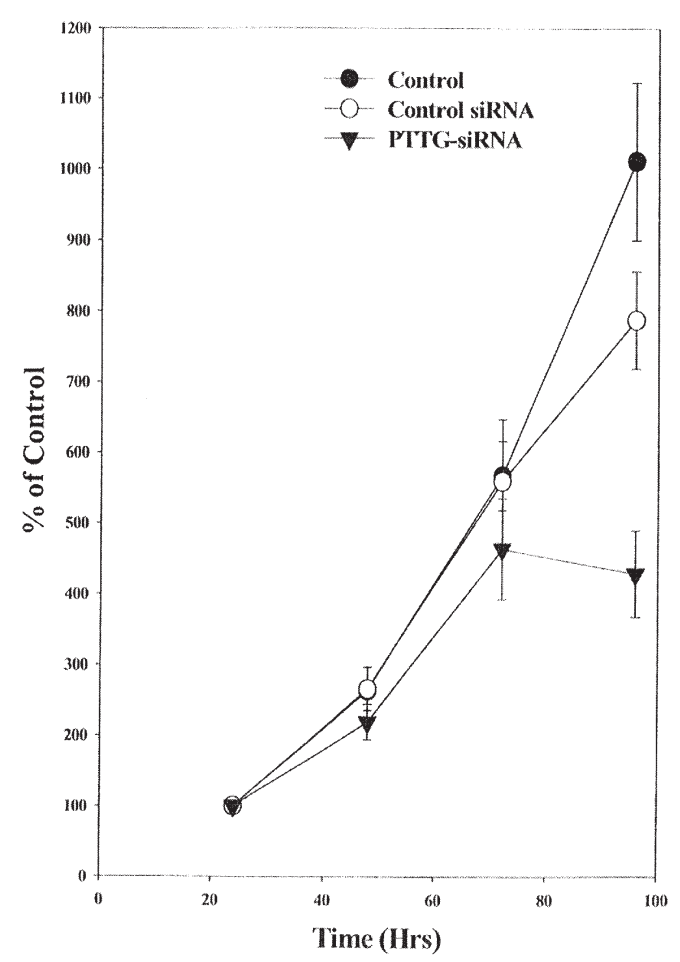

B

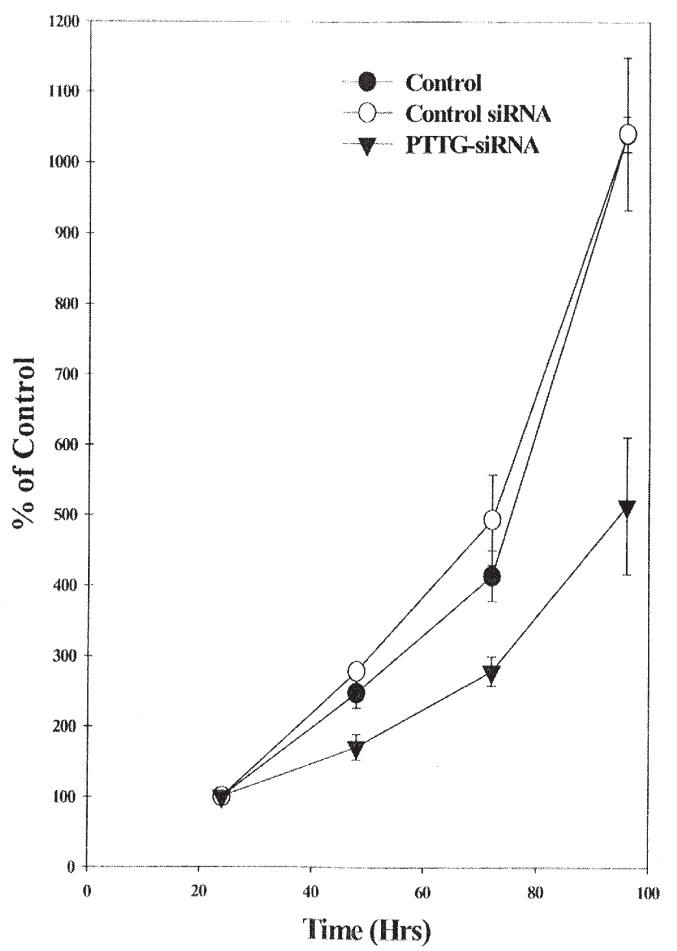

Figure 3. Effect of down-regulation of $P T T G$ on cell proliferation. Stable cells A2780 transfected with vector, control-siRNA and $P T T G$-siRNA were plated in 96-well plates and assayed for cell proliferation. A, PTTG-siRNA Clone I. B, PTTG-siRNA Clone II. A 50\% reduction in cell growth for PTTG-siRNA Clone II was observed compared to control or control-siRNA transfected cells after $72 \mathrm{~h}$ of incubation. Data shown are mean \pm SEM of three independent experiments.

endonucleases. The A2780 cells were transfected with vector, control-siRNA, or PTTG-siRNA construct. Fortyeight hours after transfection, cells were treated with $8 \mu \mathrm{g}$ of puromycin for 2 weeks and several cell lines were selected and expanded. Using Western blot analysis, we selected two cell lines showing different levels of reduction of PTTG expression; one that showed partial reduction of PTTG expression (designated as PTTG-siRNA Clone I) and another that showed complete reduction of PTTG expression (designated as PTTG-siRNA Clone II) (Fig. 2). We also selected two cell lines each from vector transfected and control siRNA transfected cells.

Effect of down-regulation of PTTG on cell proliferation. In our previous studies, we showed that overexpression of PTTG in the mouse fibroblast cell line (NIH 3T3) and human embryonic kidney cell line (HEK 293) resulted in an increase in cell proliferation, cellular transformation in vitro, and tumor development in nude mice $(8,16)$. To investigate whether down-regulation of PTTG expression contributes to cell proliferation, we compared cell proliferation of A2780 cells stably transfected with PTTG siRNA with control siRNA and vector transfected cells. Cells were seeded in quadruplicate and allowed to attach overnight, were washed in serum-free medium, and then incubated with medium containing 5\% FBS. Cell proliferation was determined using a non-radioactive assay (MTS assay) from Promega as described in Materials

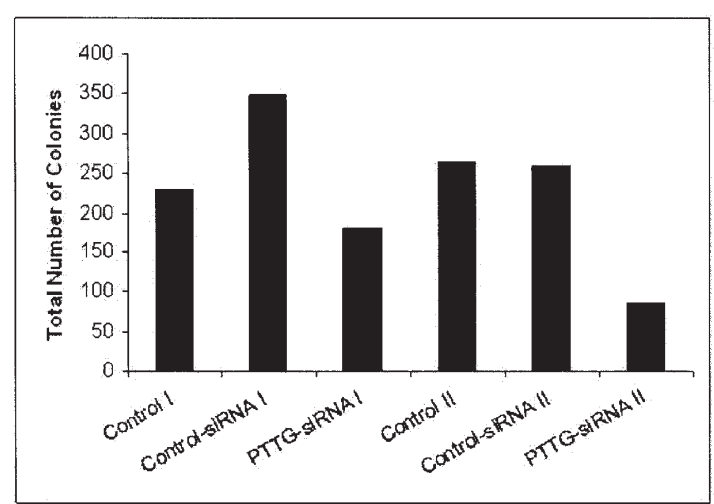

Figure 4. Effect of down-regulation of PTTG expression in A2780 cells on colony formation on soft agar. Cells were plated on soft agar as described in Materials and methods. After 12 days of plating, colonies were counted. Data shown represent from two independent experiments. A significant (70\%) reduction in colony formation was observed in PTTG-siRNA Clone II cells compared to control, control-siRNA and PTTG-siRNA Clone I cells.

and methods. As shown in Fig. 3, PTTG-siRNA Clone I did not show a significant change in cell proliferation compared to control siRNA or vector transfected cells. In contrast PTTG-siRNA Clone II showed a significant reduction in cell proliferation compared to control siRNA or vector transfected cells (Fig. 3). Previous studies from our lab and 


\section{A Control II}

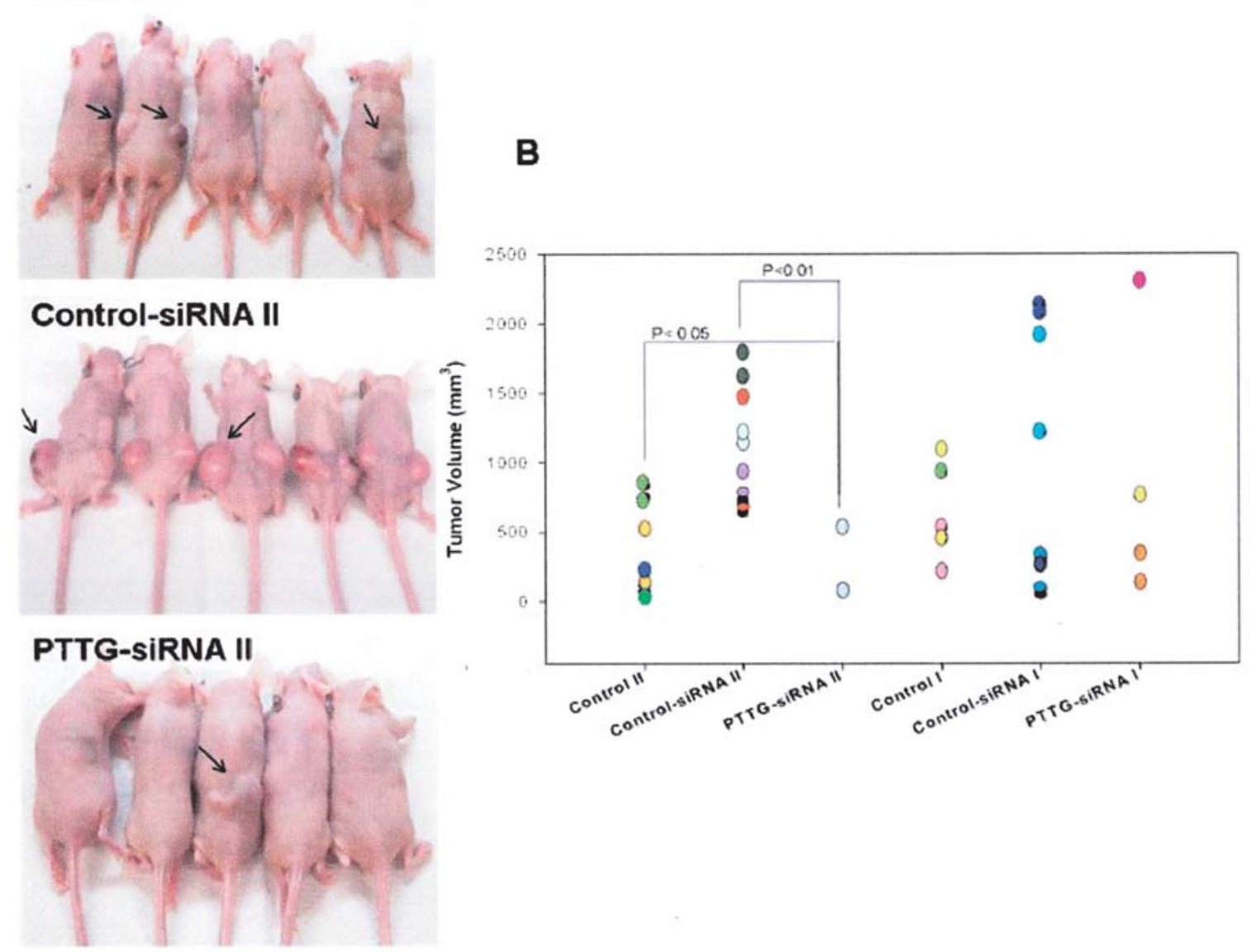

Figure 5. Down-regulation of PTTG expression in ovarian tumor cells results in suppression of tumor growth and development of tumors in nude mice. Nude mice were injected with cells stably transfected with vector, control-siRNA or PTTG-siRNA. After four weeks of injections, when large tumors were developed in animals were sacrificed (A). Tumors volumes were measured (B). PTTG-siRNA transfected cells that showed complete reduction of PTTG expression showed significantly reduced tumor growth and incidence of tumor development. Out of 5 animals, only 1 animal developed tumors. Animals injected with cells transfected with control siRNA showed larger tumors than control vector transfected cells. Reason for such changes remains unclear.

others showed that down-regulation of PTTG expression by transient transfection of tumor cells with antisense oligonucleotide $(22,23)$, PTTG siRNA (6), or PTTG siRNA adenovirus (24) reduced cell proliferation of ovarian cervical cancer cells (HelaS3), ovarian cancer cells (SK-OV3), lung cancer cells (H1299), and hepatoma cells (SH-J1), respectively. Consistent with these results, Melmed and colleagues showed reduced cell proliferation of pancreatic $\beta$ cells (26) in PTTG knockout animals, suggesting the importance of PTTG in cell proliferation.

Down-regulation of PTTG expression in A2780 cells results in inhibition of colony formation in soft agar. A soft agar growth assay was used to analyze the effect of PTTG downregulation on the anchorage-independent property of A2780 cells stably transfected with PTTG siRNA. Ten thousand cells from each cell line were plated in soft agar and allowed to grow for 12 days. A $70 \%$ reduction in the total number of colonies was detected in PTTG-siRNA Clone II (Fig. 4). These results are consistent with our results obtained with the H1299 lung tumor cell line (6), confirming that downregulation of PTTG expression is capable of reversing the transformation ability of PTTG in vitro.

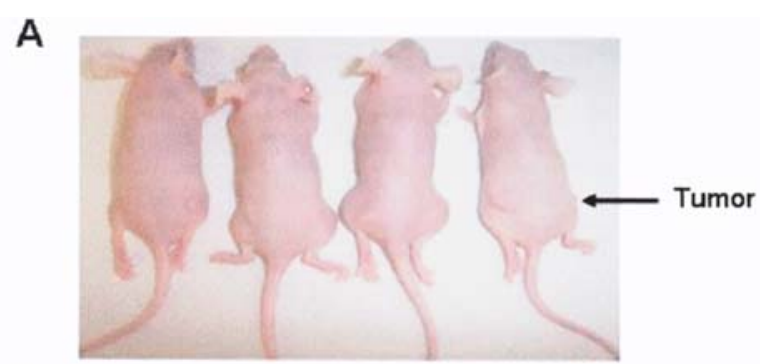

B

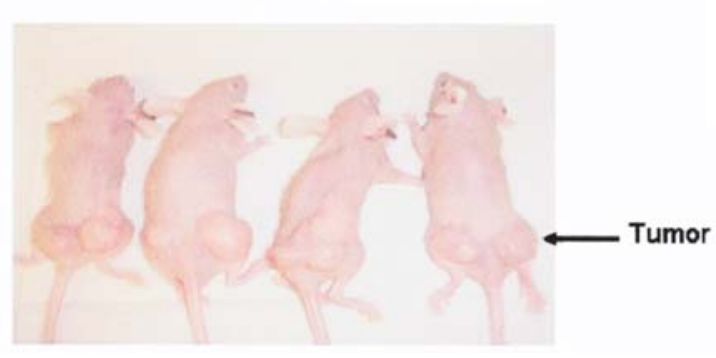

Figure 6. Effect of depletion of PTTG in HCT116 cells on tumor development and growth. HCT116 wild-type and PTTG knockout HCT116/PTTG (-/-) cells were injected into nude mice s.c. After four weeks of injection when large tumors were developed, animals were sacrificed and analyzed for tumor growth. Depletion of PTTG in HCT116 cells resulted in reduction in incidence of tumor development and tumor growth. A, HCT116/PTTG (-/-) cells. B, HCT116 wild-type cells that express high levels of PTTG. 
Down-regulation of expression of PTTG abrogates tumor formation in nude mice. In our previous studies, we showed that overexpression of PTTG in NIH3T3 and HEK293 cells resulted in tumor development in nude mice $(8,16)$. Consistent with these results, Melmed and his colleagues recently showed that overexpression of PTTG in pituitary in transgenic animals using the $\alpha \mathrm{GSU}$ promoter leads to enlargement of the pituitary, development of pituitary adenomas, and hyperplasia of the prostate (27). In our recent studies using ovarian epithelial cell-specific expression of PTTG with an MISIIR promoter in transgenic animals, we showed enlargement of the ovary, development of glandular hyperplasia, and cystic dilation of the endometrium (unpublished results). In contrast, knockdown of PTTG results in hypoplasia of the testis and spleen, and hyperplasia of thymus (20). Furthermore, knockout of PTTG resulted in a reduction of pituitary tumor development from 87 to $30 \%$ in PTTG (-/-)/Rb (+/-) animals compared to $\mathrm{Rb}(+/-)$ animals (21), suggesting an important role of PTTG in tumorigenesis. Therefore, we sought to determine whether the tumorigenic potential of ovarian tumor cells (A2780) in vivo would be affected by down-regulation of PTTG. We undertook standard xenograft experiments in athymic nude mice. A2780 cells stably transfected with vector, control siRNA, or PTTG siRNA were transplanted to both flanks of 5 to 6 week-old mice and tumor formation was monitored over a period of 4 weeks as described previously (6). Control siRNA transfected or vector transfected cells produced large tumors in all the animals. Whereas PTTG-siRNA Clone II seems to have lost this tumorigenic potential (Fig. 5). Out of 5 animals injected with cells transfected with PTTG-siRNA Clone II, only 1 animal developed tumors at the site of injection. However, tumor volume in these animals was comparatively smaller than tumors developed in animals injected with vector transfected or control siRNA transfected cells. In contrast, animals injected with PTTG-siRNA Clone I developed large tumors similar to control-siRNA or vector transfected cells. These results suggest that complete, but not the partial reduction of PTTG expression in cancer cells is required to reverse the cancer phenotypes. To confirm our findings, we used HCT116 and HCT116/PTTG (-/-) (28) cells injected s.c. into nu/nu animals. Similar to A2780 cells transfected with vector or control siRNA, HCT116 wild-type cells that express high levels of PTTG (28) developed large tumors, whereas PTTG knockout HCT116/PTTG (-/-) cells failed to develop tumors or developed small tumors (Fig. 6). Taken together, these data indicate high levels of PTTG expression are necessary for proliferation of A2780 cells and tumor development in vivo. These results are consistent with Cho-Rok et al (24), who showed suppression of tumor development in nude mice after down-regulation of PTTG expression in hepatoma cells on infection with PTTG siRNA adenovirus.

In conclusion, our results clearly demonstrate that downregulation of PTTG in ovarian tumor cells (A2780) results in the inhibition of cell proliferation and colony formation in vitro and tumor development in vivo. Based on these results, we conclude that PTTG may serve as a potential molecular target for the development of small molecules that can either inhibit the expression of PTTG or block its tumorigenic function to treat cancer. Based on the tendency of patients to develop resistance to chemotherapeutic agents, understanding of PTTG down-regulation holds the potential to serve as a novel strategy and an alternative to existing treatments.

\section{Acknowledgements}

Authors are thankful to Mr. Andrew Marsh for his help in editing the manuscript. This work was supported in part by a grant from NCI CA82511(SSK) and funds from Kentucky Lung Cancer Program (SSK).

\section{References}

1. Dutta T, Sharma H, Kumar L, Dinda AK, Kumar S, Bhatla N and Singh N: Neoadjuvant chemotherapy for epithelial ovarian cancer-role of apoptosis. Cancer Chemother Pharmacol 56: 427-435, 2005.

2. Villedieu M, Deslandes E, Duval M, Heron JF, Gauduchon P and Poulain L: Acquisition of chemoresistance following discontinuous exposures to cisplatin is associated in ovarian carcinoma cells with progressive alteration of FAK, ERK and p38 activation in response to treatment. Gynecol Oncol 101: 507-519, 2006.

3. Pei L and Melmed S: Isolation and characterization of a pituitary tumor-transforming gene (PTTG). Mol Endocrinol 11: 433-441, 1997.

4. Heaney AP and Melmed S: Pituitary tumour transforming gene: a novel factor in pituitary tumour formation. Best Pract Res Clin Endocrinol Metab 13: 367-380, 1999.

5. Zhang X, Horwitz GA, Prezant TR, Valentini A, Nakashima M, Bronstein MD and Melmed S: Structure, expression, and function of human pituitary tumor-transforming gene (PTTG). Mol Endocrinol 13: 156-166, 1999.

6. Kakar SS and Malik MT: Suppression of lung cancer with siRNA targeting PTTG. Int J Oncol 29: 387-395, 2006.

7. Heaney AP, Singson R, McCabe CJ, Nelson V, Nakashima M and Melmed S: Expression of pituitary-tumour transforming gene in colorectal tumours. Lancet 355: 716-719, 2000.

8. Kakar SS and Jennes L: Molecular cloning and characterization of the tumor transforming gene (TUTR1): a novel gene in human tumorigenesis. Cytogenet Cell Genet 84: 211-216, 1999.

9. Kakar SS, Chen L, Puri R, Flynn SE and Jennes L: Characterization of a polyclonal antibody to human pituitary tumor transforming gene 1 (PTTG1) protein. J Histochem Cytochem 49: 1537-1546, 2001

10. Puri R, Tousson A, Chen L and Kakar SS: Molecular cloning of pituitary tumor transforming gene 1 from ovarian tumors and its expression in tumors. Cancer Lett 163: 131-139, 2001.

11. Zou H, McGarry TJ, Bernal T and Kirschner MW: Identification of a vertebrate sister- chromatid separation inhibitor involved in transformation and tumorigenesis. Science 285: 418-422, 1999.

12. Romero F, Multon MC, Ramos-Morales F, Dominguez A, Bernal JA, Pintor-Toro JA and Tortolero M: Human securin, hPTTG, is associated with $\mathrm{Ku}$ heterodimer, the regulatory subunit of the DNA-dependent protein kinase. Nucleic Acids Res 29: 1300-1307, 2001

13. Wang Z and Melmed S: Pituitary tumor transforming gene (PTTG) transforming and transactivation activity. J Biol Chem 275: 7459-7461, 2000.

14. McCabe CJ, Boelaert K, Tannahill LA, Heaney AP, Stratford AL, Khaira JS, Hussain S, Sheppard MC, Franklyn JA and Gittoes NJ: Vascular endothelial growth factor, its receptor KDR/Flk-1, and pituitary tumor transforming gene in pituitary tumors. J Clin Endocrinol Metab 87: 4238-4244, 2002.

15. Ishikawa H, Heaney AP, Yu R, Horwitz GA and Melmed S Human pituitary tumor-transforming gene induces angiogenesis J Clin Endocrinol Metab 86: 867-874, 2001

16. Hamid T, Malik MT and Kakar SS: Ectopic expression of PTTG1/securin promotes tumorigenesis in human embryonic kidney cells. Mol Cancer 4: 3, 2005.

17. Christopoulou L, Moore JD and Tyler-Smith C: Overexpression of wild-type Securin leads to aneuploidy in human cells. Cancer Lett 202: 213-218, 2003.

18. Kim D, Pemberton H, Stratford AL, Buelaert K, Watkinson JC, Lopes V, Franklyn JA and McCabe CJ: Pituitary tumour transforming gene (PTTG) induces genetic instability in thyroid cells. Oncogene 24: 4861-4866, 2005. 
19. Solbach C, Roller M, Fellbaum C, Nicoletti M and Kaufmann M: PTTG mRNA expression in primary breast cancer: a prognostic marker for lymph node invasion and tumor recurrence. Breast 13: 80-81, 2004.

20. Wang Z, Yu R and Melmed S: Mice lacking pituitary tumor transforming gene show testicular and splenic hypoplasia, thymic hyperplasia, thrombocytopenia, aberrant cell cycle progression, and premature centromere division. Mol Endocrinol 15: 1870-1879, 2001.

21. Chesnokova V. Kovacs K. Castro AV. Zonis S and Melmed S: Pituitary hypoplasia in Pttg-/- mice is protective for $\mathrm{Rb}+/$ - pituitary tumorigenesis. Mol Endocrinol 19: 2371-2379, 2005.

22. Chen G, Li J, Li F, Li X, Zhou J, Lu Y and Ma D: Inhibitory effects of anti-sense PTTG on malignant phenotype of human ovarian carcinoma cell line SK-OV-3. J Huazhong University of Science and Technology. Med Sci 24: 369-372, 2004.

23. Solbach C, Roller M, Peters S, Nicoletti M, Kaufmann M and Knecht R: Pituitary tumor- transforming gene (PTTG): a novel target for anti-tumor therapy. Anticancer Res 25: 121-125, 2005.
24. Cho-Rok J, Yoo J, Jang YJ, Kim S, Chu IS, Yeom YI, Choi JY and Im DS: Adenovirus-mediated transfer of siRNA against PTTG1 inhibits liver cancer cell growth in vitro and in vivo. Hepatology 43: 1042-1052, 2006.

25. Novina CD and Sharp PA: The RNAi revolution. Nature 430: 161-164, 2004.

26. Wang Z, Moro E, Kovacs K, Yu R and Melmed S: Pituitary tumor transforming gene-null male mice exhibit impaired pancreatic beta cell proliferation and diabetes. Proc Natl Acad Sci USA 100: 3428-3432, 2003.

27. Abbud RA, Takumi I, Barker EM, Ren SG, Chen DY, Wawrowsky K and Melmed S: Early multipotential pituitary focal hyperplasia in the alpha-subunit of glycoprotein hormonedriven pituitary tumor-transforming gene transgenic mice. Mol Endocrinol 19: 1383-13891, 2005.

28. Jallepalli PV, Waizenegger IC, Bunz F, Langer S, Speicher MR, Peters JM, Kinzler KW, Vogelstein B and Lengauer C: Securin is required for chromosomal stability in human cells. Cell 105: 445-457, 2001. 